\title{
Acessibilidade dos sítios web dos governos estaduais brasileiros: uma análise quantitativa entre 1996 e 2007 *
}

\author{
André Pimenta Freire** \\ Mário de Castro*** \\ Renata Pontin de Mattos Fortes****
}

SumÁrio: 1. Introdução; 2. Acessibilidade na web e governo eletrônico; 3. Metodologia para avaliação da acessibilidade dos sítios web dos governos estaduais brasileiros; 4. Resultados das avaliações; 5. Conclusões.

Summary: 1. Introduction; 2. Web accessibility and e-government; 3. Method for evaluating the accessibility of the Brazilian state government websites; 4. Evaluation results; 5 . Conclusions.

Palavras-chave: acessibilidade na web; governo eletrônico; inclusão digital; análise quantitativa; métricas de acessibilidade.

KeY wORDs: web accessibility; e-government; digital inclusion; quantitative analysis; accessibility metrics.

A utilização da web para a disponibilização de informações e serviços de órgãos governamentais para os cidadãos tem se tornado cada vez mais expressiva. Assim, a

\footnotetext{
* Artigo recebido em abr. e aceito em dez. 2008. Os autores agradecem ao CNPq pelo apoio financeiro para o desenvolvimento do trabalho que deu origem a este artigo.

** Mestre em ciências da computação pelo Instituto de Ciências Matemáticas e de Computação da Universidade de São Paulo (ICMC/USP); pesquisador do Projeto Tidia-Ae da Fapesp no Laboratório Intermídia - ICMC/USP. Endereço: Av. do Trabalhador São-carlense, 400 - Centro - Caixa Postal 668 - CEP 13560-970, São Carlos, SP, Brasil. E-mail: apfreire@gmail.com.

**** Doutor em estatística pelo Instituto de Matemática e Estatística da Universidade de São Paulo (IME/USP); professor doutor do Departamento de Matemática Aplicada e Estatística do ICMC/USP. Endereço: Av. do Trabalhador São-carlense, 400 - Centro - Caixa Postal 668 - CEP 13560-970, São Carlos, SP, Brasil. E-mail: mcastro@icmc.usp.br.

$* * * *$ Doutora em física computacional pelo Instituto de Física de São Carlos da Universidade de São Paulo (IFSC/USP); professora associada do Departamento de Ciências de Computação do ICMC/USP. Endereço: Av. do Trabalhador São-carlense, 400 - Centro - Caixa Postal 668 CEP 13560-970, São Carlos, SP, Brasil. E-mail: renata@icmc.usp.br.
} 
garantia de que esses conteúdos e serviços possam ser acessíveis a qualquer cidadão é imprescindível, independentemente de necessidades especiais ou de quaisquer outras barreiras. No Brasil, o Decreto-Lei no 5.296/2004 determinou que todos os órgãos governamentais deveriam adaptar seus sítios na web de acordo com critérios de acessibilidade até dezembro de 2005. Com o objetivo de verificar a evolução da acessibilidade ao longo dos anos e como foi o impacto dessa legislação, este artigo analisa a acessibilidade dos sítios dos governos estaduais brasileiros por meio de amostras coletadas entre 1996 e 2007. Foram efetuadas análises por meio de métricas, obtidas por avaliações com ferramentas automáticas. Os resultados indicam que a legislação teve pouco impacto para a melhoria real da acessibilidade dos sítios no período indicado, com uma melhora somente em 2007. Verifica-se que se faz necessário adotar políticas públicas mais efetivas para que as pessoas com necessidades especiais tenham os seus direitos para acesso a informações e aos serviços públicos na web assegurados mais amplamente.

\section{Accessibility of Brazilian state government websites: a quantitative analysis between 1996 and 2007}

The use of the web to provide government information and services to citizens has become more and more significant. Ensuring that any citizen can access these contents and services is essential, regardless of disabilities or other barriers they may have. In Brazil, Executive Act no. 5.296/2004 ruled that all government agencies should adapt their websites according to accessibility guidelines until December 2005. In order to check the trend of accessibility over the years and the impact of such legislation, this article analyzes the accessibility of the Brazilian state government websites from 1996 to 2007. Analyses were carried out by means of metrics, obtained by evaluations with automatic tools. The results indicate that the legislation has had little impact on the accessibility of the sites, with an improvement in 2007. Thus, it indicates that it is necessary to adopt more effective public policies to guarantee that people with disabilities have insured their rights to access information and public services on the web.

\section{Introdução}

O uso da web ${ }^{1}$ para disponibilização de informações para os cidadãos nas diversas esferas da administração pública tem se ampliado com enorme rapidez. Cada vez mais, órgãos dos governos federal, estaduais e municipais têm utilizado a web para prover diversos tipos de serviços para os cidadãos (Rezende, 2007).

\footnotetext{
${ }^{1}$ Adotamos o termo web para referenciar a world wide web, por ser amplamente disseminado.
} 
As possibilidades que a web oferece para que esses serviços estejam ao alcance dos diversos cidadãos são do interesse de todos. Entretanto, para garantir que qualquer cidadão realmente possa ter acesso a essas informações e serviços, é necessário levar em conta diversas questões, como a disponibilidade de infraestrutura de comunicação e os meios tecnológicos utilizados para a disponibilização dos conteúdos.

Nesse quesito, a acessibilidade se apresenta como um aspecto essencial para promover a inclusão na sociedade da informação. Em particular, observar a acessibilidade em conteúdos disponíveis nos sítios públicos e governamentais na web é fundamental para garantir acesso participativo e universal do cidadão brasileiro ao conhecimento, independente de deficiências ou qualquer outra barreira.

Em 2004, a promulgação do Decreto-Lei o 5.296 marcou a primeira determinação por vias legais sobre a necessidade da observância de questões de acessibilidade em portais e sítios governamentais.

O conhecimento sobre as implicações para o desenvolvimento do conteúdo na web, da legislação de acessibilidade brasileira, ainda é reduzido no Brasil, segundo estudos recentes realizados pelo primeiro autor deste artigo (Freire, 2008). De acordo com um levantamento realizado em 2007, com mais de 600 participantes de todo o país, entre pessoas envolvidas com desenvolvimento para web em órgãos do governo, academia e indústria, mais de $40 \%$ afirmaram nunca ter ouvido falar da legislação, e $32 \%$ afirmaram só ter ouvido falar ou conhecer vagamente.

Pesquisas têm mostrado que a acessibilidade nos conteúdos dos sítios e portais governamentais ainda não está totalmente de acordo com as recomendações de acessibilidade, apesar da determinação do Decreto-Lei no 5.296/2004 de que esses conteúdos deveriam ter sido adaptados até dezembro de 2005 (Ferreira et al., 2007).

Apesar da existência de avaliações sobre portais específicos e do conhecimento sobre os problemas com acessibilidade desses sítios, ainda não existem estudos amplos e fundamentados em dados quantitativos que demonstrem o estado atual da acessibilidade em sítios governamentais no Brasil.

Neste artigo foi realizado um estudo sobre a acessibilidade dos sítios dos governos estaduais do Brasil por meio da obtenção de métricas. Foram utilizadas amostras de versões das páginas nos últimos nove anos, entre 1996 e 2007. A partir da obtenção de dados quantitativos, por meio de métricas de acessibilidade, foi possível efetuar análises estatísticas e verificar a evolução da acessibilidade dos sítios, bem como realizar testes estatísticos a fim de ve- 
rificar a aderência à legislação em momentos específicos do período estudado na linha do tempo.

Este artigo está organizado da seguinte forma: na seção 2 são apresentados conceitos fundamentais relacionados à acessibilidade na web e suas implicações no âmbito do governo eletrônico; na seção 3 vemos a metodologia utilizada para a pesquisa realizada; na seção seguinte são apresentados e discutidos os principais resultados obtidos na pesquisa; e, na última seção, sintetizamos as principais conclusões e propostas de trabalhos futuros.

\section{Acessibilidade na web e governo eletrônico}

A world wide web foi concebida com o principal intuito de fornecer uma tecnologia para disponibilização de conteúdo em um formato padrão simples e poderoso, através de informações apresentadas como hipertexto, utilizando a linguagem HTML (W3C, 1999). Desde a concepção da web, Tim Berners Lee ${ }^{2}$ destacou que "o poder da web está em sua universalidade. Ser acessada por todos, independentemente de deficiência, é um aspecto essencial".

Com o desenvolvimento e difusão da web, diversas tecnologias que não seguem padrões foram criadas para estender as possibilidades da linguagem HTML, e grande parte dos criadores de páginas e empresas que desenvolvem navegadores deixaram de seguir os principais padrões que garantiam a universalidade da web. A não utilização desses padrões e princípios que acompanham a motivação original da criação da web trouxe diversas consequências para sua evolução, uma vez que a utilização da web por usuários com softwares não convencionais, e mesmo para o uso da web em dispositivos móveis, é dificultada pela falta de padronização.

O avanço das novas tecnologias de computação móvel e ubíqua que utilizam a web, assim como os avanços sociais para inclusão das pessoas com necessidades especiais têm colocado o tema de acessibilidade na web em destaque nos ambientes acadêmicos, empresariais e governamentais.

Acessibilidade na web corresponde a possibilitar que qualquer usuário, utilizando qualquer agente (software ou hardware que recupera e exibe conteúdo web), possa entender e interagir com o conteúdo disponível nos sítios web. Acessibilidade incorpora ainda a ideia de que todas as pessoas têm o direito de ser incluídas na sociedade, independente de deficiências,

\footnotetext{
${ }^{2}$ Inventor da web e diretor do World Wide Web Consortium (W3C).
} 
localização geográfica, barreiras de linguagem, ou outro fator (Thatcher et al., 2002).

Durante o desenvolvimento de um sítio (website é o termo usado em inglês) na web é necessário que não se leve em conta só os usuários que utilizam tecnologias similares às utilizadas pelo desenvolvedor. É preciso ter em mente que a web pode ser usada em diferentes contextos por pessoas que, por exemplo (W3C, 1999a):

v sejam incapazes de ver, ouvir, se deslocar, ou interpretar determinados tipos de informações;

v tenham dificuldade em ler ou compreender textos;

v não tenham um teclado ou mouse, ou não sejam capazes de utilizá-los;

$\checkmark$ possuam tela que apresenta apenas texto, ou com dimensões reduzidas, ou ainda uma conexão lenta com a internet;

v não falem ou compreendam fluentemente o idioma em que o documento foi escrito;

V estejam com seus olhos, mãos ou ouvidos ocupados (por exemplo, ao volante, a caminho do trabalho, ou em um ambiente barulhento);

v possuam uma versão ultrapassada de navegador web, diferente dos habituais, um navegador por voz, ou um sistema operacional pouco convencional.

A partir desses exemplos, pode-se verificar que o tema "acessibilidade na web" não diz respeito somente ao acesso à web por usuários com deficiências. A utilização dos padrões e recomendações de acessibilidade traz benefícios para muitos outros grupos de usuários, e para a web como um todo (Hull, 2004).

Em 2000, 14,5\% da população brasileira possuía algum tipo de necessidade especial, e 9,6\% da população tinha mais de 60 anos de idade. Especialmente essa parcela da população de indivíduos com mais de 60 anos é bastante substancial, e representa uma grande porção de potenciais clientes de sítios de comércio eletrônico, usuários de serviços do governo, visitantes de sítios de entretenimento, entre outros. Assim, a preocupação com acessibilidade na web deve ser ainda maior em um futuro próximo, pois a parcela da população idosa, por exemplo, está crescendo expressivamente com o aumento da expectativa de vida. Segundo projeções do IBGE (IBGE, 2005), estima-se que em 2030 cerca de $20 \%$ da população mundial terá mais de 65 anos. 
Também se deve considerar que existe uma estreita relação entre a faixa etária e o surgimento de deficiências. A proporção de pessoas portadoras de necessidades especiais com menos de 21 anos é de $10 \%$, enquanto para pessoas com mais de 65 anos essa proporção é de 47,3\% (IBGE, 2005).

No cenário mundial, diversos esforços têm sido dedicados para se aperfeiçoar métodos de suporte ao desenvolvimento de aplicações web acessíveis. Além disso, diretrizes têm sido propostas para facilitar o desenvolvimento de interfaces acessíveis. O principal documento referente a diretrizes de acessibilidade é o WCAG - Web content accessibility guidelines (W3C, 1999a) do W3C - World Wide Web Consortium.

Os primeiros países que idealizaram parâmetros de acessibilidade na internet foram Canadá, Estados Unidos e Austrália, em 1997. Nos Estados Unidos, em 1998, entrou em vigor a "section 508", uma lei determinando que a tecnologia eletrônica e de informação dos órgãos federais seja acessível às pessoas com necessidades especiais. Segundo essa lei, a tecnologia inacessível interfere na capacidade individual de adquirir e usar a informação de maneira rápida e fácil. A section 508 foi decretada para eliminar barreiras na tecnologia da informação, disponibilizando novas oportunidades para as pessoas com necessidades especiais e incentivando o desenvolvimento de tecnologias que as auxiliem a atingir esses objetivos (US Government, 1998).

Com o objetivo de tornar a web acessível a um número cada vez maior de pessoas e maximizar a interoperabilidade, o W3C criou a iniciativa para a acessibilidade na web (web accessibility initiative, WAI). Entre outras atribuições, essa WAI mantém grupos de trabalho para elaborar conjuntos de recomendações para garantir a acessibilidade do conteúdo da web às pessoas com necessidades especiais ou que acessam a web em condições especiais de ambiente, equipamento, navegador e outras ferramentas web. Como resultado desse trabalho, em 1999 foi publicada a primeira versão das recomendações para a acessibilidade do conteúdo da web (WCAG 1.0, W3C, 1999a), principal referência mundial em termos de acessibilidade na web.

Além dessa iniciativa, também em 1999, Portugal regulamentou a adoção de regras de acessibilidade à informação disponibilizada na internet pela administração pública para pessoas com necessidades especiais. Essa iniciativa transformou Portugal no primeiro país da Europa e o quarto no mundo a legislar sobre acessibilidade na web. Em 2000, ao aprovar o plano de ação eEurope 2002 (que inclui o compromisso da adoção das recomendações sobre acessibilidade do W3C nos sítios públicos), o Conselho Europeu estendeu a iniciativa portuguesa aos 15 países da União Europeia. 
No Brasil, o modelo de acessibilidade de governo eletrônico (e-MAG) (Governo brasileiro, 2008) foi elaborado pelo Ministério do Planejamento, Orçamento e Gestão para a construção e a adaptação dos conteúdos governamentais (informações, serviços etc.) na internet. Nesse modelo são fornecidas: uma visão do cidadão (conceitual) e uma visão técnica (na forma de cartilha) com recomendações fundamentadas no conjunto de regras do W3C, mas com indicações simplificadas e priorizações adaptadas à realidade e às necessidades brasileiras. Essas recomendações visam que o processo para tornar os conteúdos do governo brasileiro acessíveis seja conduzido de forma padronizada, de fácil implementação, coerente com as necessidades brasileiras, e em conformidade com os padrões internacionais. Esse modelo é a referência de todas as instituições governamentais brasileiras para a construção e adaptação das suas soluções de governo eletrônico com interface web. O e-MAG foi criado especificamente para atender ao Decreto-Lei no $5.296 / 2004$, que regulamenta as Leis nos $10.048 / 2000$ e 10.098/2000.

A avaliação da acessibilidade de portais de governo eletrônico no Brasil foi o alvo de um trabalho de pesquisa desenvolvido por Ferreira e colaboradores (2007). No trabalho, foi efetuada a avaliação automática de 351 sítios da administração pública ou de interesse público em três momentos: em novembro de 2005 (um mês antes do prazo para finalizar as adaptações, de acordo com o Decreto-Lei no 5.296/2004), em março de 2006 e em março de 2007. As avaliações foram efetuadas por meio da ferramenta daSilva (DaSilva, 2008).

No estudo, verificou-se que em novembro de 2005 apenas cinco sítios foram classificados com o conceito AAA (atende aos níveis de prioridade 1, 2 e 3) de acordo com o WCAG do W3C e somente quatro com conceito AAA de acordo com o e-MAG. Nos testes realizados em março de 2006, apenas um sítio obteve conceito AAA. Em março de 2007, 22 sítios foram classificados com conceito AAA.

De acordo com os dados do estudo, pôde-se verificar que poucas instituições estavam em observância da legislação brasileira de acessibilidade. O estudo de Ferreira e colaboradores (2007) apresentou dados relevantes sobre a acessibilidade nos sítios governamentais brasileiros. Contudo, a forma como a avaliação foi efetuada não permitiu fazer análises estatísticas mais apuradas.

Em pesquisa anterior, Freire e colaboradores (2007) investigaram sobre as formas de processamento automático para obtenção de dados quantitativos que possibilitassem monitorar a acessibilidade de sítios web. Essa pesquisa realizou coleta de informações de uma amostra de páginas de sítios de governos municipais brasileiros e propôs um método prático para o processamento 
do levantamento de dados e respectivo cálculo das métricas relativas a atendimento dos critérios de acessibilidade.

Neste artigo, a proposta para avaliação se restringe a um cenário mais específico, dos governos estaduais. A avaliação proposta envolve o uso de métricas de acessibilidade, que permitem que análises mais apuradas sejam efetuadas. Além disso, também é feita a análise da acessibilidade com amostras dos sítios desde 1996 até 2007.

\section{Metodologia para avaliação de acessibilidade dos sítios web dos governos estaduais brasileiros}

O objetivo principal da realização deste artigo foi o de verificar a evolução da acessibilidade dos sítios web dos governos estaduais entre 1996 e 2007. Em dezembro de 2004, com a promulgação do Decreto-Lei no 5.296/2004, estabeleceu-se um prazo de 12 meses para que todos os portais governamentais fossem adequados para acomodar requisitos de acessibilidade. Assim, também foi proposta a verificação da hipótese de que essa determinação tenha sido realmente cumprida ou não no prazo estipulado.

Assim, propôs-se como abordagem para verificar a adequação dos sítios dos governos estaduais a utilização de métodos de avaliação nas versões das páginas em diferentes anos, com a análise quantitativa dos resultados.

Para recuperar as versões antigas das páginas, foi utilizada a ferramenta way back machine (Internet Archive, 2008) do Projeto Internet Archive. No projeto, foram feitas amostragens de páginas de toda a internet em diferentes dias, desde 1996. Essas páginas foram armazenadas em um servidor para posterior recuperação.

$\mathrm{Na}$ análise, para a coleta da amostra para cada ano utilizou-se a primeira ocorrência válida do sítio de cada governo estadual. Para o ano de 2007, utilizou-se a versão do dia $1^{\circ}$ de setembro de 2007 obtida diretamente pelo sítio web de cada estado, pois, até a data da realização, as amostras do ano de 2007 não estavam disponíveis por meio do way back machine.

Para obter avaliações mais precisas, além da avaliação da página principal de cada sítio, também foram avaliadas todas as páginas que se encontravam no nível de hierarquia imediatamente abaixo da página principal. Logo, um total de 1.232 páginas foram avaliadas, correspondendo a uma média de 45,63 páginas por sítio. A ferramenta HTTrack (HTTrack, 2008) foi utilizada para efetuar a varredura dos links nas páginas e para fazer cópia de todos os arquivos das páginas a serem avaliadas. Essa etapa levou aproximadamente 10 horas para completar o processamento. 
Normalmente, na maioria das avaliações de acessibilidade de sítios web encontradas na literatura, utiliza-se a classificação por níveis A, AA ou AAA, do WCAG (W3C, 1999a). Nessa classificação, temos que:

- o nível A indica que todos os pontos de verificação de prioridade 1 (significando prioridade mais importante, cujos pontos devem ser observados) foram atendidos;

v no nível AA, todos os pontos de prioridade 1 e 2 foram atendidos;

v no nível AAA, todos os pontos de prioridade 1, 2 e 3 foram atendidos na avaliação.

Contudo, verifica-se que tal avaliação não fornece um ferramental adequado para fazer comparações mais apuradas dos níveis de acessibilidade. Por exemplo, existem diversos sítios que não atendem sequer ao nível A, e também existe bastante variação entre sítios dentro de um mesmo nível.

Dessa forma, neste artigo, propôs-se a utilização de métricas de acessibilidade, que calculam o número de problemas de acessibilidade em relação ao número de problemas em potencial. Por exemplo, se uma imagem pode representar um problema de acessibilidade caso não tenha um texto alternativo (que pode prejudicar a utilização por uma pessoa com deficiência visual), ela representa um problema em potencial.

Além do cálculo da razão entre problemas de acessibilidade em relação ao número de problemas em potencial, também são utilizados pesos para diferenciar problemas com diferentes níveis de prioridade.

A expressão utilizada para calcular as medidas de acessibilidade foi a da métrica WAB (web accessibility barrier, Parmanto e Zenj, 2005). A seguir, é ilustrado o cálculo da métrica, onde $N P$ é o número total de páginas do sítio; $B_{b p}$ é o número de barreiras do tipo $b$ encontradas em uma página $p ; P_{b p}$ é o número de barreiras em potencial do tipo $b$ encontradas em uma página $p$; e $W_{b}$ é o peso associado a cada tipo de barreira, de acordo com o inverso do nível de prioridade de cada barreira definido pelo WCAG (W3C, 1999a). Assim, pontos com níveis de prioridade 1 têm peso 1 , com prioridade 2 têm peso $1 / 2$ e com prioridade 3 têm peso 1/3.

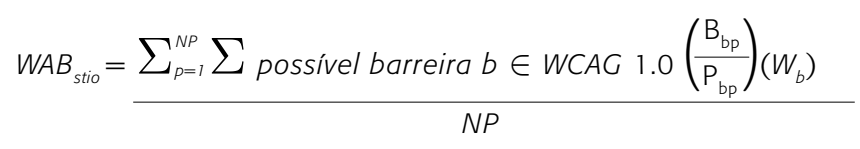


Para efetuar a avaliação do grande número de páginas coletadas foram utilizadas ferramentas automáticas. Vale observar que tais ferramentas não são capazes de avaliar todas as diretrizes e pontos de verificação de acessibilidade, uma vez que muitos deles só podem ser identificados a partir de uma avaliação efetuada manualmente. Contudo, os pontos avaliados por ferramentas automáticas podem fornecer importantes indicativos dos problemas de acessibilidade.

No quadro são apresentados os pontos de verificação do WCAG (W3C, 1999a) que foram possíveis de ser utilizados nas avaliações automáticas. Os pontos de verificação (PV) estão dispostos da maneira que se apresentam nas diretrizes (D). Também são identificados os níveis de prioridade de cada um deles.

\section{Pontos de verificação de acessibilidade do WCAG, avaliados automaticamente}

Diretrizes (D) e pontos de verificação (PV)

D 1: prover alternativas equivalentes para conteúdo audiovisual.

PV 1.1: fornecer um equivalente textual a cada elemento não-textual.

PV 1.5: fornecer links textuais redundantes para cada região ativa dos mapas de imagem no cliente.

D 3: utilizar linguagem de marcação e folhas de estilo e fazê-lo de maneira apropriada.

PV 3.2: criar documentos passíveis de validação por gramáticas formais, publicadas.

PV 3.3: utilizar folhas de estilo para controlar a paginação (disposição em página) e a apresentação.

PV 3.4: utilizar unidades relativas, e não absolutas, nos valores dos atributos da linguagem de marcação e nos valores das propriedades das folhas de estilo.

PV 3.5: utilizar elementos de cabeçalho indicativos da estrutura do documento, de acordo com as especificações.

D 4: simplificar o uso de linguagem natural.

PV 4.3: identificar o principal idioma utilizado nos documentos.

D 5: criar tabelas, que tipos de tabela? Passíveis de transformação harmoniosa.

PV 5.5: fornecer resumos das tabelas.

D 6: garantir que páginas com novas tecnologias tenham transformação harmoniosa.

PV 6.2: assegurar que os equivalentes de conteúdo dinâmico sejam atualizados sempre que esse conteúdo mudar.

PV 6.3: assegurar que todas as páginas possam ser utilizadas mesmo que os programas interpretáveis, os applets ou outros objetos programados tenham sido desativados ou não sejam suportados.

\section{Prioridade}

1

3

2

2

2

2

3

1

1

Continua 
Diretrizes (D) e pontos de verificação (PV)

Prioridade

PV 6.5: assegurar que todas as páginas possam ser utilizadas mesmo que os 2 programas interpretáveis, os applets ou outros objetos programados tenham sido desativados ou não sejam suportados.

D 7: garantir o controle do usuário sobre mudanças de conteúdo temporizadas.

PV 7.4: não criar páginas de atualização automática periódica, até que os agentes do usuário possibilitem parar essa atualização.

PV 7.5: não utilizar marcações para redirecionar as páginas automaticamente, até que os agentes do usuário possibilitem parar o redirecionamento automático.

D 9: projetar pensando na independência de dispositivos.

PV 9.5: fornecer atalhos por teclado que apontem para links importantes.

D 10: utilizar soluções de transição.

PV 10.2: assegurar o posicionamento correto de todos os controles de formulários que tenham rótulos implicitamente associados.

PV 10.4: incluir caracteres predefinidos de preenchimento nas caixas de edição e nas áreas de texto, até que os agentes do usuário tratem corretamente os controles vazios.

PV 10.5: inserir, entre links adjacentes, caracteres que não funcionem como link e sejam passíveis de impressão (com um espaço de início e outro de fim).

D 11: utilize tecnologias e diretrizes do W3C.

PV 11.1: utilizar tecnologias do W3C sempre disponíveis e adequadas a uma determinada tarefa; utilizar as versões mais recentes, desde que suportadas.

PV 11.2: evitar funcionalidades desatualizadas de tecnologias do W3C.

D 12: fornecer informações de orientação e de contexto.

PV 12.1: dar, a cada frame, um título que facilite a identificação dos frames e sua navegação.

PV 12.2: Descrever a finalidade dos frames e o modo como se relacionam entre si, se isso não for óbvio a partir unicamente dos títulos.

PV 12.3: Dividir grandes blocos de informação em grupos mais fáceis de gerenciar, sempre que for o caso.

PV 12.4: associar explicitamente os rótulos aos respectivos controles.

Utilizando a fórmula $\mathrm{WAB}$, o valor máximo de índice de barreiras de acessibilidade que poderia ser atingido é de 12,5. Deve-se ressaltar que um índice de barreiras baixo não indica necessariamente que os sítios estejam completamente acessíveis, mas que não apresentam grande número de problemas em relação aos pontos de verificação que podem ser verificados automaticamente.

Para automatizar o cálculo das métricas, foram analisadas diferentes ferramentas de avaliação de acessibilidade disponíveis. A ferramenta escolhi- 
da foi a Hera (Benavídez et al., 2006), por ser uma ferramenta de código aberto e de fácil manipulação, e por ser bastante popular entre pessoas envolvidas com desenvolvimento para web. As ferramentas brasileiras disponíveis não são de código aberto, e por isso o trabalho para adaptação para automatização dos cálculos seria bastante complicado.

Na figura 1 é ilustrada uma tela com o resultado de uma avaliação de acessibilidade efetuada pela ferramenta Hera. Na figura pode-se notar que, por exemplo, na página sob avaliação, os pontos de verificação 3.2 e 3.5, que possuem prioridade 2 , foram infringidos.

\section{Figura 1}

Exemplo de resultado parcial de avaliação de acessibilidade com a ferramenta Hera

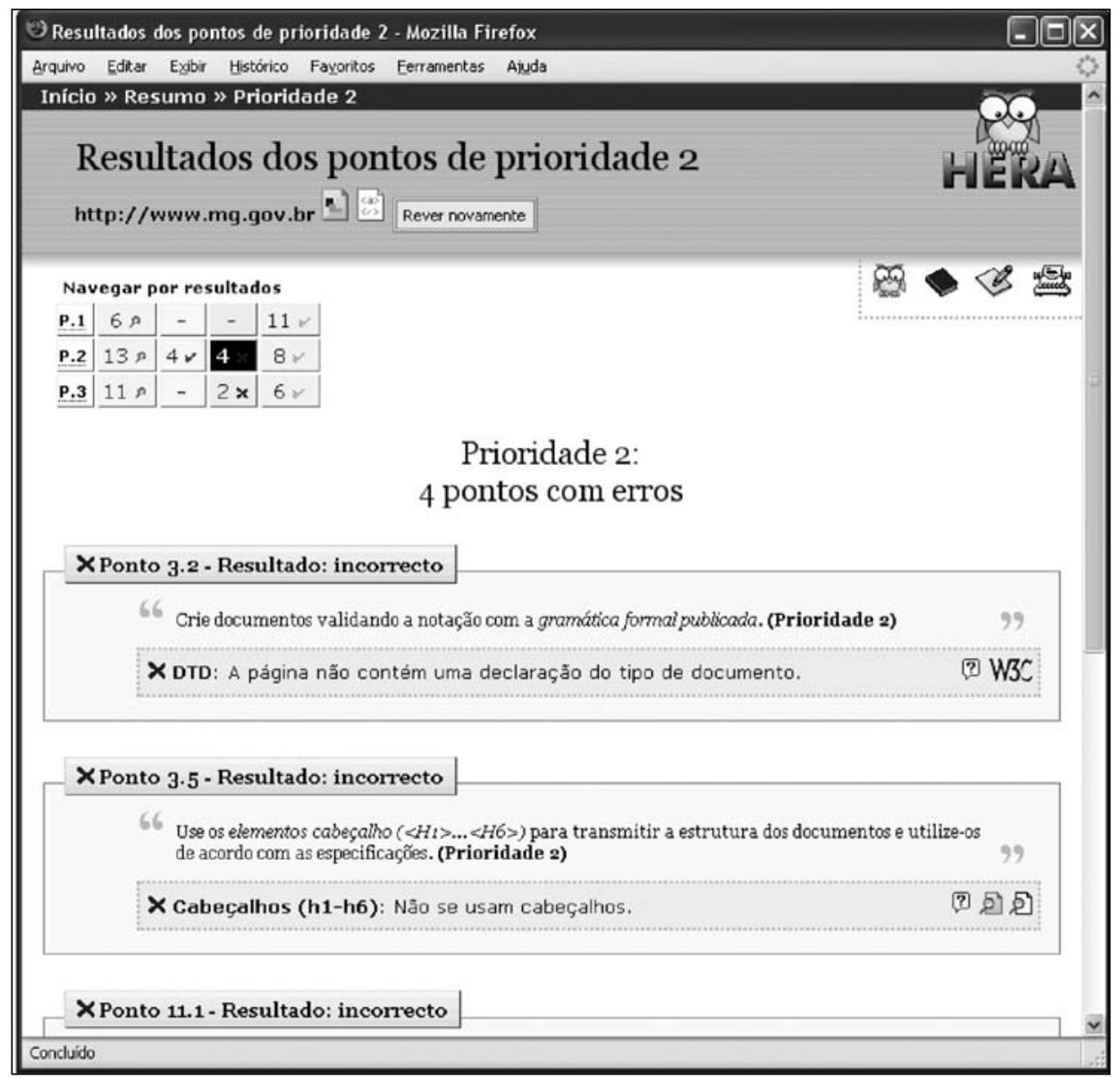


Dessa forma, com o uso dos crawlers para recuperação e amostragem das páginas e com a ferramenta Hera, foram efetuadas as avaliações utilizadas para conduzir as análises da acessibilidade dos sítios dos governos estaduais.

Na próxima seção são apresentados os principais resultados e as análises estatísticas das métricas obtidas por meio da metodologia descrita.

\section{Resultados das avaliações}

A partir do processamento das avaliações de acessibilidade efetuadas por meio da ferramenta Hera, foi efetuada uma análise estatística dos dados obtidos, por meio da observação do comportamento das métricas de acessibilidade e da realização de inferências com o objetivo de verificar se houve evolução da acessibilidade, principalmente no período entre 2004 e 2006, em que os sítios deveriam ter sido adaptados, de acordo com a legislação brasileira.

No gráfico da figura 2 é possível observar a evolução no número de estados com sítios web em cada ano. Verifica-se que, em 1996, apenas um estado (Ceará) possuía um sítio web arquivado no internet web archive. Em 1999, 12 estados já tinham sítio na web, enquanto em 2000 havia 21 estados com sítios na web. Dessa forma, verifica-se que nesse período ocorreu o maior crescimento de implantação de sítios web dos governos estaduais no Brasil. A partir de 2003, todos os estados brasileiros já possuíam sítios web.

A seguir são apresentados os gráficos com a evolução dos índices de barreiras de acessibilidade dos sítios dos governos estaduais no decorrer dos anos. Vale notar que os números altos correspondem a sítios com índices mais altos de barreiras. É importante ressaltar que um índice baixo de barreiras com essa avaliação não indica necessariamente que o sítio não tenha problemas de acessibilidade, uma vez que só foi utilizado o método de avaliação automática, que não garante cobertura total dos problemas de acessibilidade.

Nos gráficos da figura 3, com as séries históricas das avaliações com a métrica WAB, pode-se verificara evolução dos índices de barreiras dos sítios dos governos estaduais agrupados por regiões geográficas. 


\section{Figura 2}

Quantidade de unidades da Federação (UFs) com

sítios web arquivados, de acordo com o ano

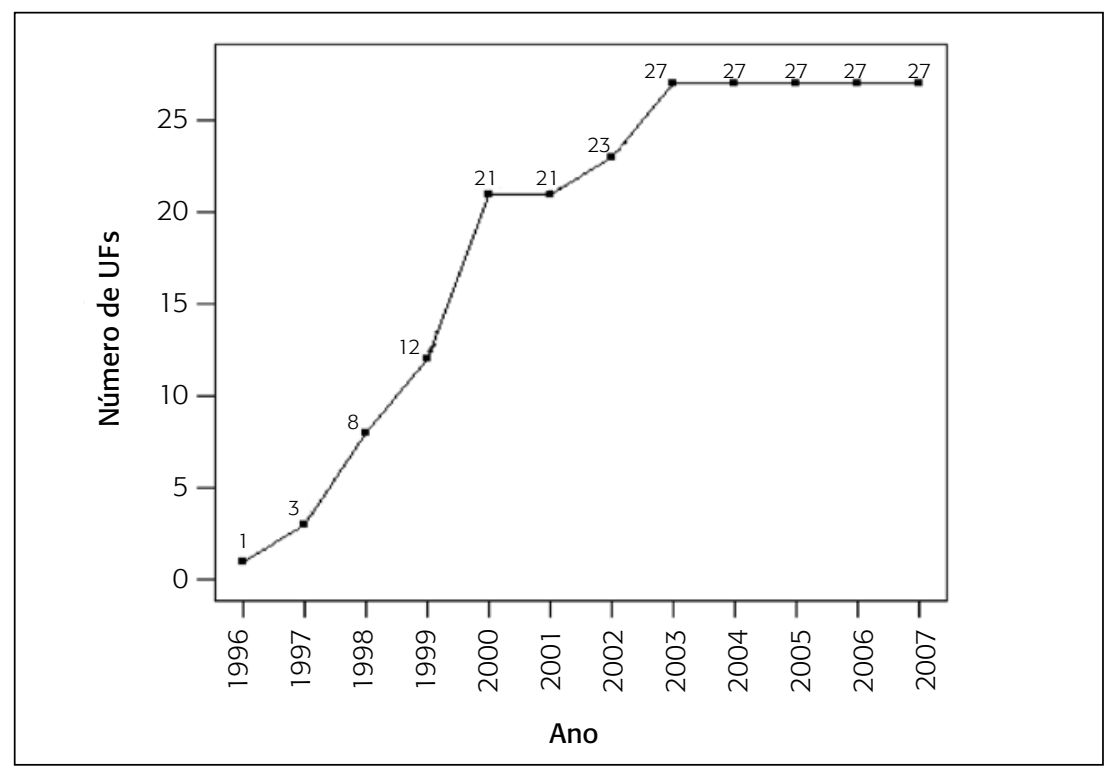

Em geral, pode-se observar que nos anos correspondentes a trocas de governos ocorrem mudanças mais perceptíveis em alguns estados, geralmente advindas de processos de reestruturação estabelecidos pelas novas equipes de governo. Observa-se também que os índices são considerados relativamente altos.

Na região Norte, verifica-se que em geral há poucas variações bruscas dos índices de barreiras de acessibilidade. Nos anos mais recentes (entre 2006 e 2007), observa-se que somente um estado (Rondônia) teve uma diminuição mais substancial do índice de barreiras de acordo com os critérios examinados pela ferramenta automática.

No caso da região Centro-oeste, observa-se uma tendência de diminuição nos índices de barreiras, que só foi mais substancial em um estado (Mato Grosso).

Na região Nordeste, que congrega o maior número de estados, observase que alguns estados tiveram diminuição no índice de barreiras de seus sítios, enquanto outros tiveram até aumento nos períodos mais recentes. Só se nota uma diminuição mais expressiva no sítio do governo do Ceará, em 2007. 


\section{Figura 3}

\section{Séries históricas da métrica WAB dos sítios web dos governos estaduais brasileiros}

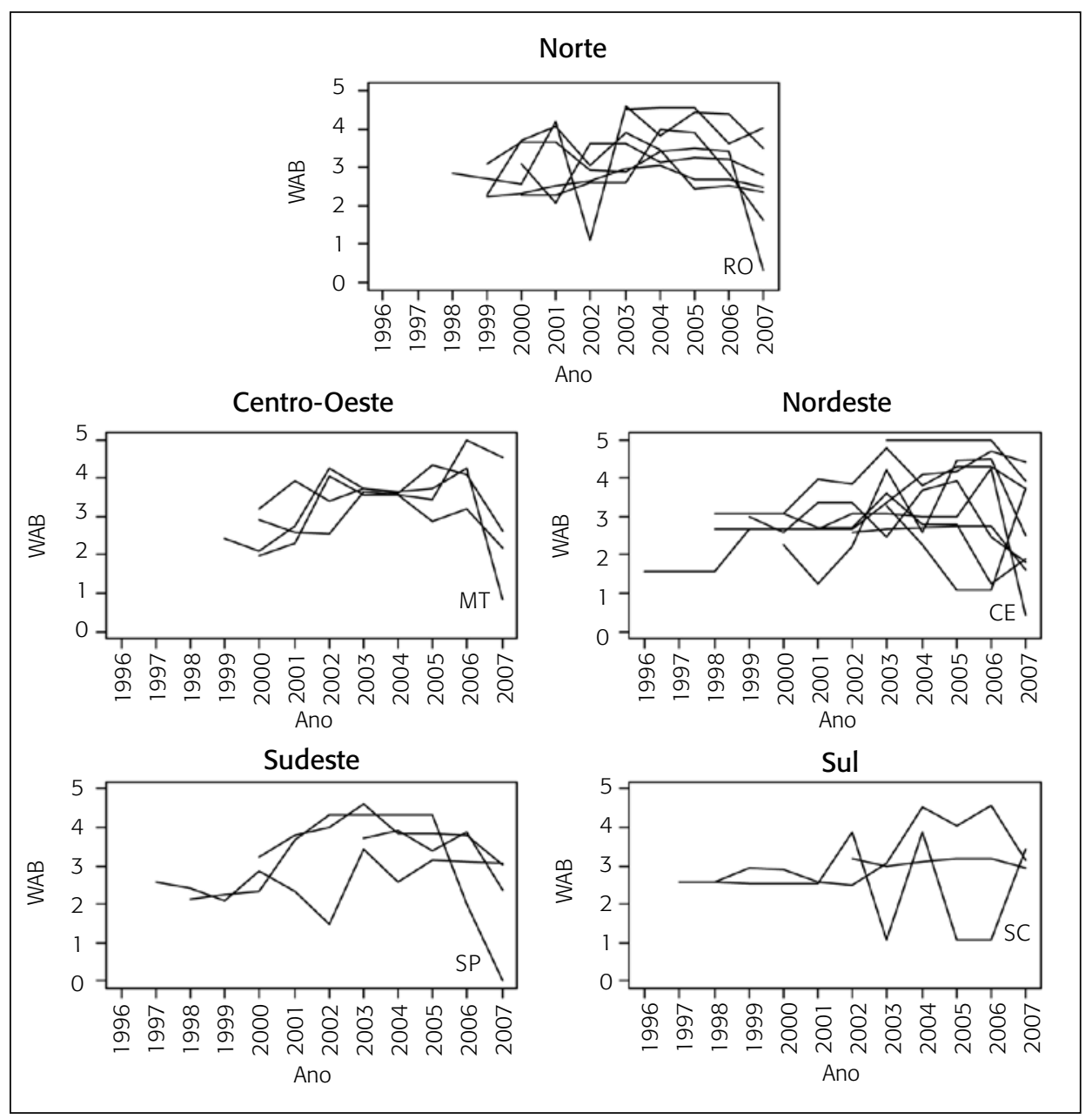

Na região Sudeste, verifica-se que nos anos mais recentes houve poucas mudanças nos índices, com exceção do estado de São Paulo, que teve uma diminuição mais substancial. Na região Sul, os índices também não tiveram grandes variações, com exceção do estado de Santa Catarina, que teve um aumento do índice de barreiras mais expressivo entre 2006 e 2007.

A partir dessa análise, pode-se ressaltar que o problema da acessibilidade nos sítios web dos governos estaduais ocorre em todas as regiões do país. 
Também é interessante observar que os sítios anteriores a 2000 em geral tinham índices de barreiras de acessibilidade menores. Uma possível explicação para o aumento no índice nos anos seguintes é o uso de novas tecnologias para interação sem o devido cuidado com a adequação para a acessibilidade.

Além das análises efetuadas por meio das séries históricas, foi efetuada análise estatística sobre os dados de acessibilidade dos sítios nos anos 2004 e 2006, período que compreende as versões imediatamente anteriores à promulgação do Decreto-Lei no 5.296/2004 e imediatamente após o prazo para adequação das páginas. Na figura 4 é exibido o gráfico de dispersão com a relação entre os índices de barreiras WAB dos sítios dos governos estaduais nos anos de 2004 (eixo das abscissas) e 2006 (eixo das ordenadas). Assim, os estados representados na parte inferior correspondem aos que tinham, em 2004, um índice de barreiras de acessibilidade maior do que em 2006. Já os estados representados na parte superior correspondem aos estados que tinham em 2006 um índice de barreiras de acessibilidade maior do que o índice de barreiras em 2004.

Figura 4

Gráfico de dispersão da métrica WAB entre os anos de 2004 e 2006

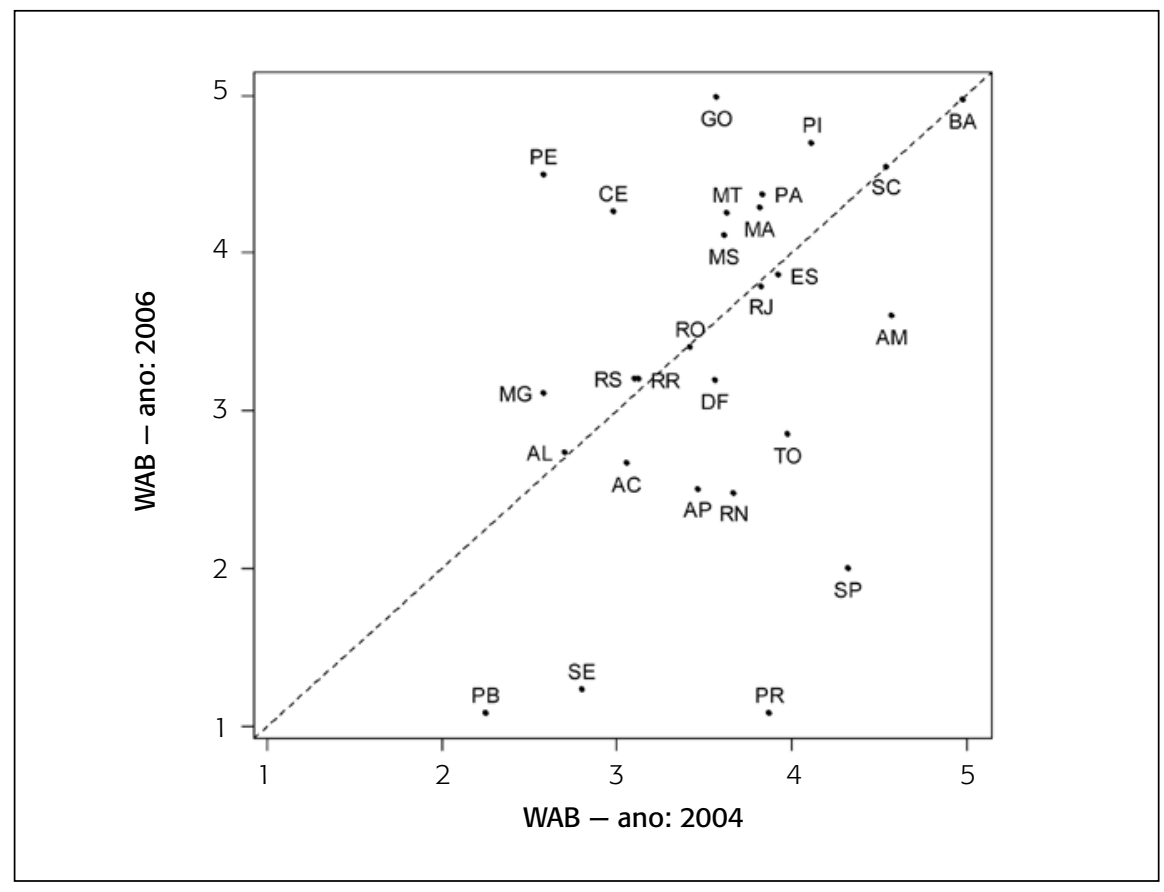


Pode-se observar no gráfico da figura 4 que grande parte dos estados não sofreu mudanças mais expressivas no índice de barreiras de acessibilidade. Muitos estados tiveram até mesmo aumento no índice de barreiras, ao invés de diminuição.

Além da análise por meio do gráfico de dispersão, também foram efetuados testes estatísticos (teste $t$ de Student para dados pareados e teste de Wilcoxon) para verificar se há indícios de mudanças significativas nos índices de barreiras.

\section{Figura 5}

Gráfico de dispersão da métrica WAB entre os anos de 2004 e 2007

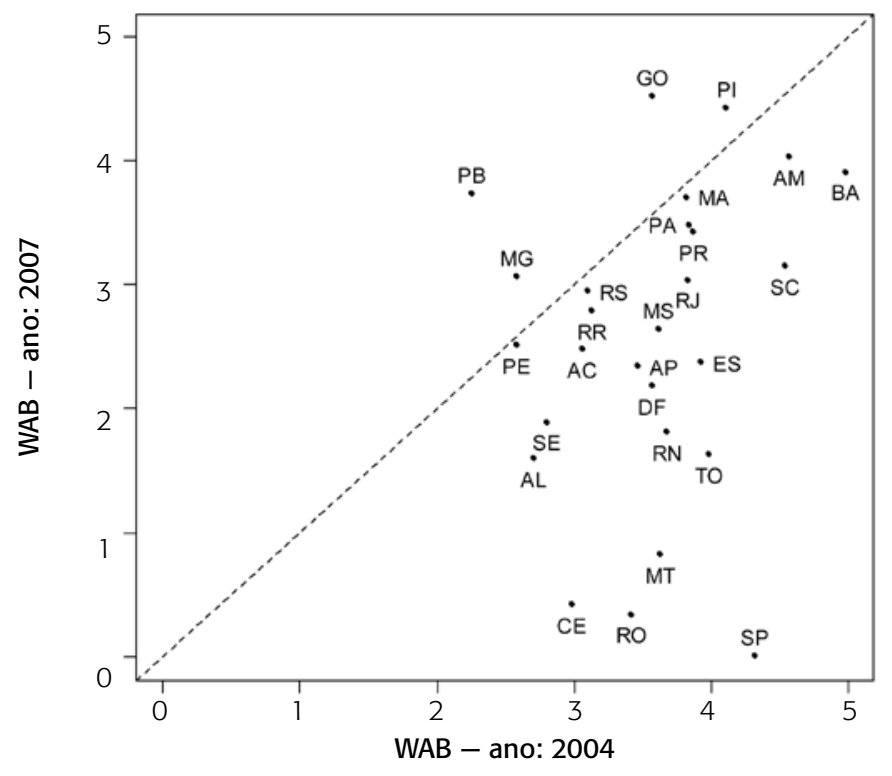

No teste $t$ obteve-se um nível descritivo $p=0,3842$, e no teste de Wilcoxon obteve-se $p=0,6790$. Com esses valores, ambos os testes indicam que, com nível de significância de 5\%, as mudanças nos valores dos índices de barreiras não foram significativas.

A partir desses resultados, somos levados a concluir que a promulgação do Decreto-Lei no 5.296/2004 não causou o efeito desejado, de diminuição dos índices de barreiras de acessibilidade nos sítios dos governos estaduais no Brasil dentro do prazo indicado. 
Contudo, com a análise entre a diferença das médias da métrica entre os anos de 2004 e 2007, pode-se notar que existem indícios de mudanças nos índices de acessibilidade, com nível descritivo $\mathrm{p}=0,0004$ no teste $t$ de Student para dados pareados e $\mathrm{p}=0,0002$ no teste de Wilcoxon.

Na figura 5, é possível observar que um número maior de estados encontra-se abaixo da linha de identidade no gráfico. Isso significa que houve um número maior de estados com diminuição no índice de barreiras em suas páginas entre 2004 e 2007.

\section{Conclusões}

A utilização da web tem se tornado cada vez mais frequente para a disponibilização de informações de órgãos públicos para os cidadãos no Brasil e em todo o mundo. Garantir que essas informações possam ser acessíveis a todas as pessoas, independentemente de deficiências, é determinante para que a democratização do acesso ao governo seja efetiva também nos meios eletrônicos.

No Brasil, verifica-se que o atendimento aos critérios de acessibilidade em sítios dos órgãos públicos ainda não é satisfatório no período estipulado pelo Decreto-Lei no 5.296, que determinava que todos os sítios governamentais deveriam ser adaptados de acordo com critérios de acessibilidade até dezembro de 2005.

Neste artigo foi efetuada uma análise da acessibilidade dos sítios dos governos estaduais das 27 unidades da Federação do Brasil, por meio de avaliação automática com cálculo de métricas quantitativas. Foram avaliadas amostras de páginas recuperadas de arquivos na internet no período entre 1996 e 2007.

A partir das análises de gráficos e de testes estatísticos, confirmou-se a hipótese de que não houve mudança significativa na acessibilidade dos sítios dos governos estaduais dentro do período estipulado, apesar das determinações da legislação. Os indicativos de mudanças e de diminuição dos índices de barreiras só foram identificados nas amostras de 2007.

Embora a maioria desses sítios na web apresente a tendência de redução no número de barreiras (figuras 4 e 5), um acompanhamento com frequência regular, amplo (incluindo avaliações por amostragem, com usuários reais) e que fosse divulgado publicamente possibilitaria melhor aproveitamento dos recursos da tecnologia de informação para a população brasileira.

Os graves problemas de acessibilidade que existem nos sítios governamentais criados para disponibilizar informações para todos os cidadãos indi- 
cam que ainda é necessário propor políticas públicas mais efetivas para garantir o acesso universal do cidadão brasileiro ao conteúdo na web.

Como trabalhos futuros, pretendemos efetuar análises mais detalhadas utilizando as diretrizes do e-MAG e abrangendo outros órgãos do governo.

\section{Referências bibliográficas}

BENAVÍDEZ, C. et al. Semi-automatic evaluation of web accessibility with Hera 2.0. In: INTERNATIONAL CONFERENCE ON COMPUTERS HELPING PEOPLE WITH SPECIAL NEEDS, 10. Proceedings... Springer, 2006. p. 199-106.

DA SILVA. Avaliador de acessibilidade daSilva. 2008. Disponível em: <www.dasilva. org.br>. Acesso em: mar. 2008.

FERREIRA, S. B. L.; SANTOS, R. C.; SILVEIRA, D. S. Panorama da acessibilidade na web brasileira. In: ENCONTRO DA ANPAD - ENANPAD, 31. Anais ... 2007. 17p.

FREIRE, A. P. Acessibilidade no desenvolvimento de sistemas Web: um estudo sobre o cenário brasileiro. 2008. Dissertação (Mestrado) — Instituto de Ciências Matemáticas e de Computação, Universidade de São Paulo, São Carlos, 2008.

; BITTAR, T. J.; FORTES, R. P. M. An approach based on metrics for monitoring web accessibility in Brazilian municipalities websites. In: ANNUAL ACM SYMPOSIUM ON APPLIED COMPUTING, 23. Fortaleza, CE, 2008. Proceedings ... New York, NY: ACM Press, 2008. v. 1. p. 2421-2425.

GOVERNO BRASILEIRO. Modelo de acessibilidade de governo eletrônico — versão 2.0. 2008. Disponível em: <www.governoeletronico.gov.br/emag/>. Acesso em: jan. 2008.

HTTRACK. Web Crawler HTTrack. Disponível em: <www.httrack.com>. Acesso em: mar. 2008.

HULL, L. Accessibility: it's not just for disabilities any more. Interactions, v. 11, n. 2, p. 36-41, 2004.

IBGE (Instituto Brasileiro de Geografia e Estatística). Censo demográfico 2000. Disponível em: <www.ibge.gov.br/censo>. Acesso em: jan. 2008.

. Síntese de indicadores sociais 2004. Estudos e pesquisas: informação demográfica e socioeconômica, 2005.

INTERNET ARCHIVE. Internet web archive. 2008. Disponível em: <www.archive. org > . Acesso em: mar. 2008. 
PARMANTO, B.; ZENG, X. Metric forweb accessibility evaluation. Journal of the American Society for Information Science and Technology, v. 56, n. 33, p. 1394-1404, 2005.

REZENDE, D. A. Planejamento de informações públicas municipais: sistemas de informação e de conhecimento, informática e governo eletrônico integrados aos planejamentos das prefeituras e municípios. Revista de Administração Pública, v. 41, n. 3, p. 505-536, maio/jun. 2007.

THATCHER, J. et al. Constructing accessible websites. Glasshaus, 2002.

US GOVERNMENT. Section 508. 1998. Disponível em: <www.section508.gov>. Acesso em: mar. 2008.

W3C, WORLD WIDE WEB CONSORTIUM. HTML 4.01 Specification. 1999. Disponível em: <www.w3.org/TR/html4/>. Acesso em: jan. 2008.

. Web content acessibility guidelines 1.0. 1999a. Disponível em: <www. w3.org/TR/WCAG10/>. Acesso em: jan. 2008. 